\title{
Erratum: Space charge dynamics of bright electron beams [Phys. Rev. ST Accel. Beams 6, 024201 (2003)]
}

Alexander W. Chao, Rainer Pitthan, Toshiki Tajima, and Dian Yeremian

(Received 9 April 2003; published 17 April 2003)

PACS numbers: $41.75 .-\mathrm{i}, 99.10 . \mathrm{Cd}$

Based on unsuccessful searches of related topics on the Web, in the library, and by contacting colleagues, we believed in our paper that our development of a self-consistent longitudinal equation, the ansatz in Eq. (8) of our paper, and the coupled envelope equations, Eqs. (14) and (28), were a new result. This is not true.

The self-consistent longitudinal parabolic distribution was first proposed by Neuffer [1]. Coupled envelope equations have been developed and analyzed by Reiser [2]. We apologize for this shortcoming and want to quote the pertinent pathbreaking original work of Neuffer and Reiser.

[1] David Neuffer, IEEE Trans. Nucl. Sci. 26, 3031 (1979).

[2] Martin Reiser, Theory and Design of Charged Particle Beams (J. Wiley and Sons, New York, 1994), particularly Chap. 5 and Eqs. (5.494) and (5.495). 